\title{
Effects of Ester Side Chain Structure on Gas Permeation Behavior of Poly(dialkylfumarate)s
}

\author{
Sung Boo ChOI, ${ }^{\dagger}$ Atsushi TaKahara, Naoyuki AmaYA, ${ }^{*}$ \\ Yoshishige Murata, ${ }^{*}$ and Tisato KAJIYAMA ${ }^{\dagger \dagger}$ \\ Department of Applied Chemistry, Faculty of Engineering, \\ Kyushu University, Hakozaki, Higashi-ku, \\ Fukuoka 812, Japan \\ * Tsukuba Research Laboratory, Nippon Oil \& Fats Co., Ltd., \\ Tokodai 5-10, Toyosato-cho, Tsukuba, \\ Ibaraki 300-26, Japan
}

(Received June 6, 1988)

\begin{abstract}
The gas permeation behavior of poly(dialkylfumarate)s (PDAF)s with various bulky ester side chains has been studied. The thermal molecular motion and intermolecular distances depended on the bulkiness of ester side groups. The permeation behavior was strongly influenced by thermal molecular motion and density of PDAFs. The magnitude of permeability for $\mathrm{O}_{2}, \mathrm{~N}_{2}$, and $\mathrm{CO}$ is in the order of $10^{-10}-10^{-9} \mathrm{~cm}^{3}(\mathrm{STP}) \cdot \mathrm{cm} \mathrm{cm}^{-1} \mathrm{~s}^{-1} \mathrm{~cm} \mathrm{Hg}^{-1}$ at $298 \mathrm{~K}$. The magnitude of separation factor of $\mathrm{O}_{2}$ to $\mathrm{CO}$ was above 10 at $298 \mathrm{~K}$ and remained high below the $\beta$ relaxation temperature.

KEY WORDS Poly(dialkylfumarate) / Permeability / Separation / Oxygen /

Nitrogen / Thermal Molecular Motion /
\end{abstract}

The separation process of gases through polymeric films has made great progress these days due to the appearance of new polymeric materials and the development of fabrication techniques. $^{1-3}$ Membrane based gas permeation plays important roles for industrial processes such as hydrocarbon recovery from purge streams ${ }^{4}$ and oxygen enrichment. ${ }^{5}$ The permeation behavior of gases across nonporous membrane is influenced by the thermal molecular motion. ${ }^{6}$ Below the glass transition temperature, $T_{\mathbf{g}}$, the membrane is in a glassy state, the molecular motion of polymer chains is restricted and the contribution from activated diffusion is very small. ${ }^{7,8}$ In the rubbery state, the free segmental motion is active so that chain mobility and diffusivity increase. In general, the permeation rate in a rubbery polymer is higher than that of a glassy one; however, a glassy polymer shows higher selectivity due to the lower molecular mobility in the glassy state.

The high temperature separation process has an advantage from the view point of energy saving. Also, the mechanical strength of membrane is an important factor. In order to keep high permselectivity at high temperature, it is necessary to develop a new class of glassy polymers. The poly(dialkylfumarate) (PDAF) is a candidate. This polymer shows a high glass transition temperature due to its rigid rod-like structure. ${ }^{9-12}$ Also, the aggregation state can be controlled by changing the bulky ester side chains.

In this study, the effects of ester side chain structure on permeation behavior of PDAFs

\footnotetext{
$\dagger$ Present address: Department of Chemical Engineering, Dongguk University, Seoul 100, Korea.

${ }^{\dagger \dagger}$ To whom correspondence should be addressed.
} 
with various ester side chain groups is discussed.

\section{EXPERIMENTAL}

\section{Materials}

The chemical structure and ester substituents of PDAFs used in this experiment are shown in Figure 1. In order to reveal the effects of ester substituents, two series of PDAFs were used in this study. One of the two ester substituents of each series of PDAFs is isopropyl alcohol (iP) or tert-butyl alcohol (tB). Other substituents are cyclohexyl alcohol $(\mathrm{cH})$, secbutyl alcohol (sB), and 4-methylpentyl alcohol $(4 \mathrm{mP})$. These polymers are designated as $\mathrm{P}(\mathrm{R}-$ iPF) or $P(R-t B F)$ where $R$ indicates the various types of ester substituents. The film specimen of these polymers was prepared by casting a $1 \mathrm{wt} \%$ benzene solution on a clean glass plate at room temperature. This film was subsequently dried in vacuo for $12 \mathrm{~h}$. The film thickness of these films was about $25 \mu \mathrm{m}$.

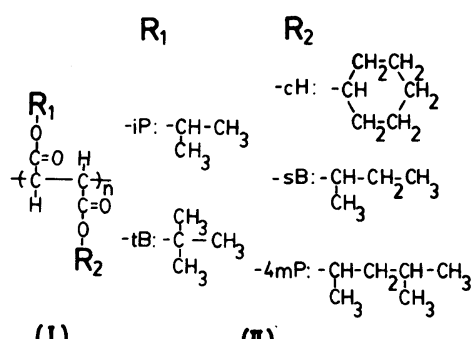

(l)

(II)

Figure 1. Chemical structures of poly(alkyl isopropylfumarate)s and poly(alkyl $t$-butylfumarate)s and their ester substituents.

Table I. Properties of polyalkylfumarates

\begin{tabular}{llrrr}
\hline \multicolumn{1}{c}{ Polymer } & \multicolumn{1}{c}{$\mathrm{R}_{1}$} & \multicolumn{1}{c}{$\mathrm{R}_{2}$} & \multicolumn{1}{c}{$\bar{M}_{n}$} & $d / \mathrm{g} \mathrm{cm}^{-3}$ \\
\hline P(cH-iPF) & -cH & -iP & 77100 & 1.124 \\
P(sB-iPF) & -sB & -iP & 89000 & 1.094 \\
P(4mP-iPF) & $-4 \mathrm{mP}$ & $-\mathrm{iP}$ & 101300 & 1.078 \\
P(iP-tBF) & -iP & -tB & 88500 & 1.084 \\
P(cH-tBF) & -cH & -tB & 49500 & 1.100 \\
P(sB-tBF) & $-\mathrm{sB}$ & -tB & 63200 & 1.076 \\
P(4mP-tBF) & $-4 \mathrm{mP}$ & -tB & 41800 & 1.038 \\
\hline
\end{tabular}

Table I summarizes the ester substituents, the number average molecular weight, and density of poly(alkyl isopropylfumarate)s and poly(alkyl $t$-butylfumarate)s used in this study.

\section{Gas Permeation Experiment}

The gases employed for the permeation experiment were $\mathrm{H}_{2}, \mathrm{~N}_{2}, \mathrm{O}_{2}$, and $\mathrm{CO}$ and had a purity above $99.9 \%$. The gas permeation measurements were carried out with $20 \mathrm{~K}$ intervals from 298 to $453 \mathrm{~K}$ by the high vacuum method. ${ }^{13} \mathrm{~A}$ stainless steel permeation cell with effective area of $0.79 \mathrm{~cm}^{2}$ was used and sealed with a Viton ${ }^{\circledR}$ gasket. The MKS Baratoron pressure transducer was used to monitor continuously the down stream pressure.

\section{RESULTS AND DISCUSSION}

\section{Permeation Behavior of Poly(dialkylfumarate)}

The permeability for various gases are shown in the form of Arrhenius plots in Figures 2 to 5 . The magnitude of permeability at $298 \mathrm{~K}$, pre-exponential factor, and the activation energy are summarized in Table II. The

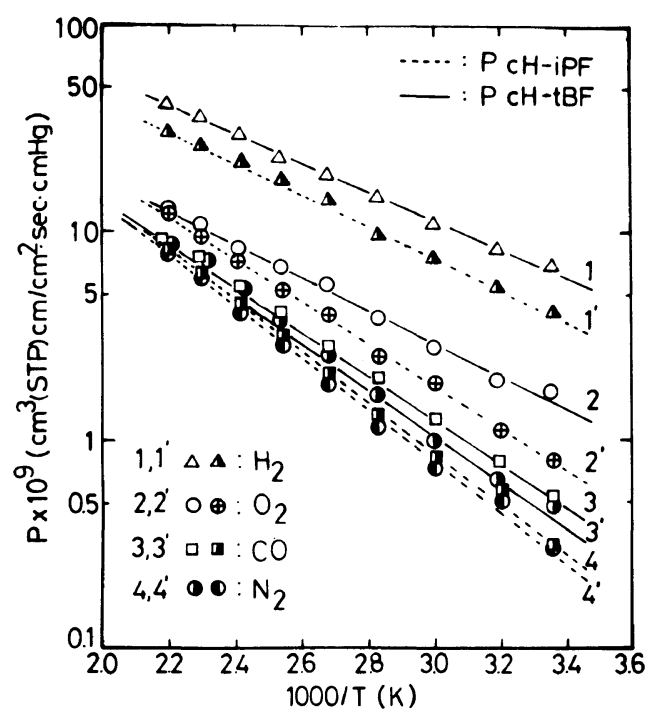

Figure 2. Temperature dependence of the gas permeability of poly(cyclohexyl isopropylfumarate) and poly(cyclohexyl $t$-butylfumarate). 


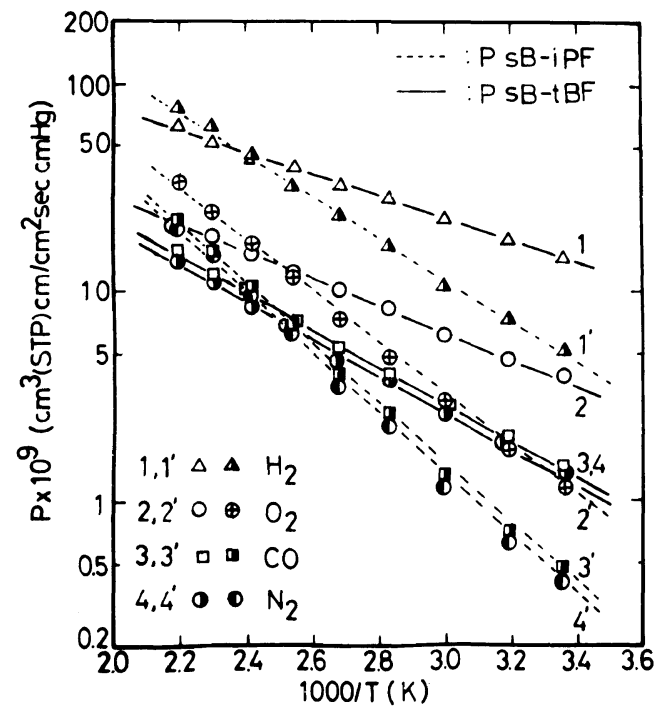

Figure 3. Temperature dependence of the gas permeability of poly(s-butyl isopropylfumarate) and poly(sbutyl $t$-butylfumarate).

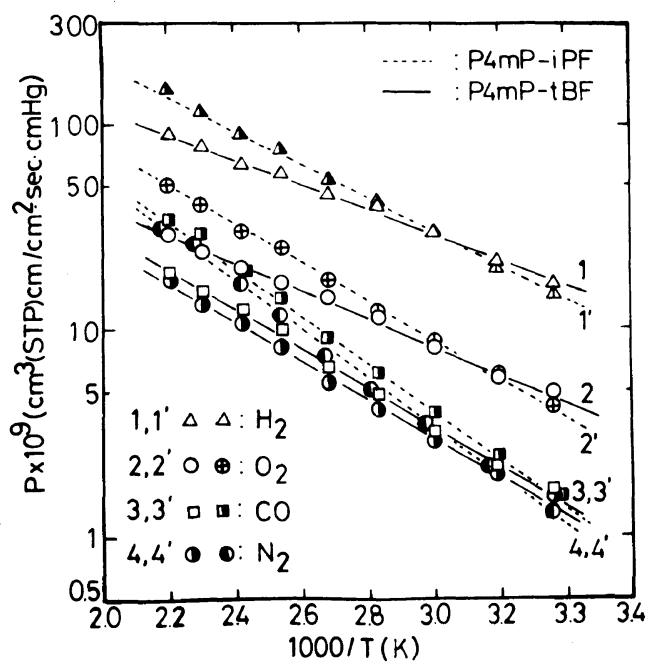

Figure 4. Temperature dependence of the gas permeability of poly(4-methylpentyl isopropylfumarate) and poly(4-methylpentyl $t$-butylfumarate).

permeability increased in the order of nitrogen, carbon monoxide, oxygen and hydrogen. There has been reported several attempts to correlate the permeability of gases with thermal molecular motion of polymeric chains. ${ }^{14-16}$ The temperature dependence of

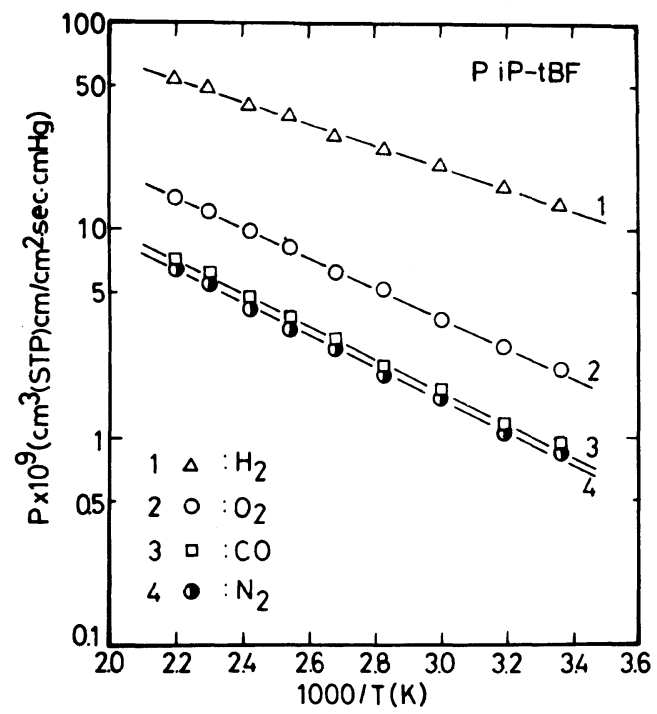

Figure 5. Temperature dependence of the gas permeability of poly(isopropyl $t$-butylfumarate).

dynamic viscoelasticity revealed that the onset of the $\beta$-relaxation for poly(alkyl $t$-butylfumarate) occurs at a lower temperature than that for poly(alkyl isopropylfumarate). ${ }^{12}$ In the case of poly(alkyl $t$-butylfumarate) with bulky $t$-butyl group, the $\beta$-relaxation occurs at a lower temperature than that for poly(alkyl isopropylfumarate). Also, the differential scanning calorimetry revealed that the thermal degradation of poly(alkyl $t$-butylfumarate) started at a lower temperature than that for poly(alkyl isopropylfumarate). This onset of thermal degradation corresponds to the $\alpha$ relaxation of PDAF. The gas permeation behavior of PDAFs can be divided into two types depending on thermal molecular motion because there is a large difference in the slopes of the Arrhenius plots of these two series. Yamada and coworkers reported that intermolecular spacing of poly(diisopropylfumarate)s increased with temperature. ${ }^{11}$ Especially the increase became remarkable at the $\beta$-absorption region which is related to the side chain motion. This steep increase in intermolecular distance will give rise to change in the diffusivity of gases. The dependence of permeability 
Table II. $P$ at $298 \mathrm{~K}, P_{0}$ and $E_{0}$ for polyalkylfumarates

\begin{tabular}{|c|c|c|c|c|c|c|}
\hline \multirow{4}{*}{ Polymer } & \multicolumn{3}{|c|}{$\mathrm{H}_{2}$} & \multicolumn{3}{|c|}{$\mathrm{O}_{2}$} \\
\hline & $P \times 10^{9}$ & $P_{0} \times 10^{6}$ & \multirow{2}{*}{$\begin{array}{c}E_{\mathrm{p}} \\
\mathrm{kcal}\end{array}$} & $P \times 10^{9}$ & $P_{0} \times 10^{6}$ & \multirow{2}{*}{$\begin{array}{c}E_{\mathrm{p}} \\
\text { kcal }\end{array}$} \\
\hline & \multicolumn{2}{|c|}{$\mathrm{cm}^{3}(\mathrm{STP}) \mathrm{cm}$} & & \multicolumn{2}{|c|}{$\mathrm{cm}^{3}(\mathrm{STP}) \mathrm{cm}$} & \\
\hline & $\mathrm{cm}^{-2} \mathrm{~s}^{-1}$ & $\mathrm{cmHg}^{-1}$ & $\mathrm{~mol}^{-1}$ & $\mathrm{~cm}^{-2} \mathrm{~s}^{-1}$ & $\mathrm{cmHg}^{-1}$ & $\mathrm{~mol}^{-1}$ \\
\hline $\mathrm{P}(\mathrm{cH}-\mathrm{iPF})$ & 4.1 & 1.3 & 3.4 & 0.8 & 2.3 & 4.7 \\
\hline $\mathrm{P}(\mathrm{sB}-\mathrm{iPF})$ & 5.1 & 14.9 & 4.8 & 1.2 & 19.8 & 5.8 \\
\hline $\mathrm{P}(4 \mathrm{mP}-\mathrm{iPF})$ & 15.6 & 9.8 & 3.9 & 4.5 & 5.0 & 4.2 \\
\hline P(iP-tBF) & 13.3 & 0.7 & 2.4 & 2.1 & 0.5 & 3.2 \\
\hline $\mathrm{P}(\mathrm{cH}-\mathrm{tBF})$ & 7.2 & 1.4 & 3.1 & 1.7 & 0.7 & 3.7 \\
\hline $\mathrm{P}(\mathrm{sB}-\mathrm{tBF})$ & 14.5 & 1.0 & 2.5 & 3.9 & 0.6 & 3.0 \\
\hline $\mathrm{P}(4 \mathrm{mP}-\mathrm{tBF})$ & 16.9 & 2.0 & 2.8 & 4.9 & 0.8 & 3.0 \\
\hline \multirow{4}{*}{ Polymer } & \multicolumn{3}{|c|}{$\mathrm{N}_{2}$} & \multicolumn{3}{|c|}{$\mathrm{CO}$} \\
\hline & $P \times 10^{9}$ & $P_{0} \times 10^{6}$ & \multirow{2}{*}{$\begin{array}{c}E_{\mathrm{p}} \\
\text { kcal }\end{array}$} & $P \times 10^{9}$ & $P_{0} \times 10^{6}$ & \multirow{2}{*}{$\begin{array}{c}E_{\mathrm{p}} \\
\mathrm{kcal}\end{array}$} \\
\hline & \multicolumn{2}{|c|}{$\mathrm{cm}^{3}(\mathrm{STP}) \mathrm{cm}$} & & \multicolumn{2}{|c|}{$\mathrm{cm}^{3}(\mathrm{STP}) \mathrm{cm}$} & \\
\hline & $\mathrm{cm}^{-2} \mathrm{~s}^{-1}$ & $\mathrm{cmHg}^{-1}$ & $\mathrm{~mol}^{-1}$ & $\mathrm{~cm}^{-2} \mathrm{~s}^{-1}$ & $\mathrm{cmHg}^{-1}$ & $\mathrm{~mol}^{-1}$ \\
\hline $\mathrm{P}(\mathrm{cH}-\mathrm{iPF})$ & 0.3 & 4.3 & 5.7 & 0.3 & 4.0 & 5.6 \\
\hline $\mathrm{P}(\mathrm{sB}-\mathrm{iPF})$ & 0.5 & 26.0 & 6.5 & 0.6 & 2.7 & 6.5 \\
\hline$P(4 m P-i P F)$ & 1.4 & 13.1 & 5.5 & 1.5 & 13.9 & 5.4 \\
\hline P(iP-tBF $)$ & 0.9 & 0.3 & 3.5 & 0.9 & 0.4 & 3.6 \\
\hline $\mathrm{P}(\mathrm{cH}-\mathrm{tBF})$ & 0.5 & 2.5 & 5.2 & 0.5 & 2.2 & 5.0 \\
\hline $\mathrm{P}(\mathrm{sB}-\mathrm{tBF})$ & 1.3 & 1.3 & 4.0 & 1.4 & 1.5 & 4.2 \\
\hline$P(4 m P-t B F)$ & 1.6 & 1.7 & 4.2 & 1.7 & 2.3 & 4.3 \\
\hline
\end{tabular}

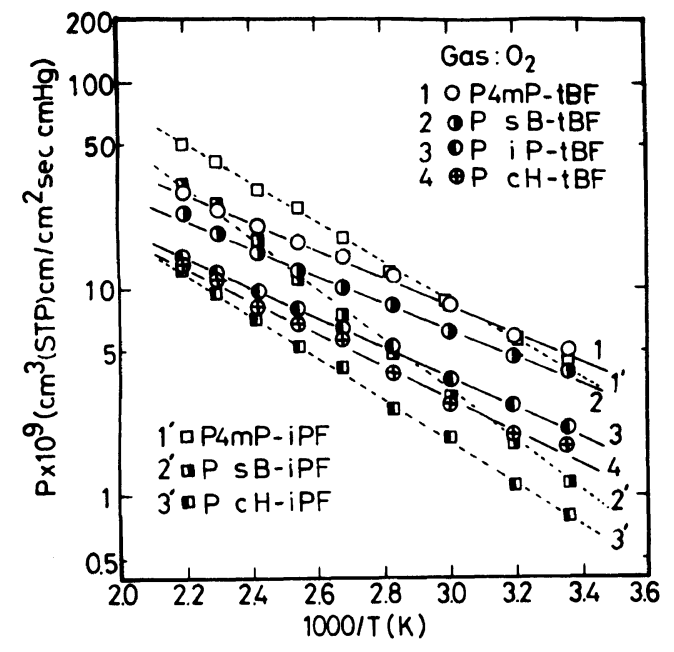

Figure 6. Temperature dependence of the permeability of oxygen through various poly(dialkylfumarate) membrane. on temperature for poly(alkyl isopropylfumarate) is larger than that for poly(alkyl $t$ butylfumarate). This reflects the state of thermal molecular motion in this temperature region. Since the bulkiness of the side chain of poly(alkyl isopropylfumarate) is smaller than that of poly(alkyl $t$-butylfumarate), a more vigorously activated side chain motion of the $\beta$-relaxation process for poly(alkyl isopropylfumarate) was observed in this temperature region.

Figure 6 displays the temperature dependences of the permeability of oxygen in poly(alkyl isopropylfumarate) and poly(alkyl $t$ butylfumarate) with various ester side groups. This difference in permeation behavior may be due to the difference in thermal molecular 


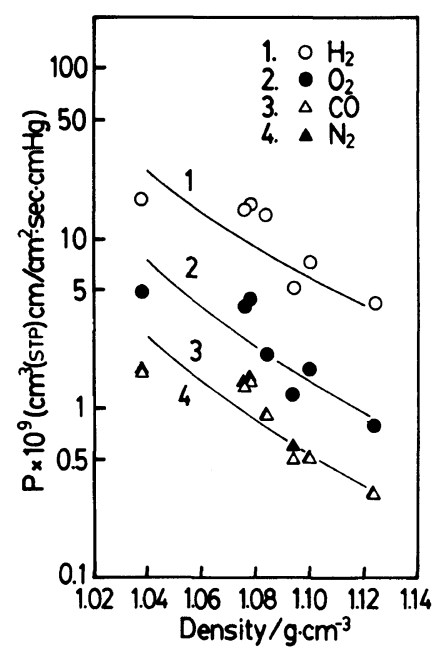

Figure 7. Relationships between gas permeability and density for poly(dialkylfumarate)s.

motion as mentioned above. The magnitude of permeability for oxygen are $1-5 \times 10^{-9}$ $\mathrm{cm}^{3}$ (STP) $\mathrm{cm} \mathrm{cm}^{-2} \mathrm{~s}^{-1} \mathrm{~cm} \mathrm{Hg}^{-1}$ at $298 \mathrm{~K}$. Compared with other glassy polymers such as poly(phenyleneoxide) (PPO) ${ }^{16}$ and polyimide, ${ }^{17}$ this magnitude is higher in PDAFs. This would be related to the aggregation state of PDAF which consists of rigid rod-like molecules.

Figure 7 illustrates the correlation between permeability of gas at $298 \mathrm{~K}$ and density of PDAF membrane. The density of each specimen is shown in Table I. The general trend is that the permeability of gases decreases with an increase in density. Despite the bulkiness of the side chains, poly(cyclohexyl isopropylfumarate), and poly(cyclohexyl $t$-butylfumarate) have high density, which might be due to the compact packing state of the side chain groups in these membranes. The decrease in permeability with increasing density suggests a large contribution of diffusivity of gases to the permeation behavior.

Since the permeability is also a function of solubility, the permeation behavior of PDAFs can be correlated with the solubility parameter calculated from chemical structure. ${ }^{18}$ Table III summarizes the solubility parameters, $\delta_{\mathrm{sp}}$ for
Table III. Solubility parameters for polyalkylfumarates

\begin{tabular}{cc}
\hline & $\delta_{\mathrm{sp}}$ \\
\cline { 2 - 2 } & $\left(\mathrm{cal} \mathrm{cm}^{-3}\right)^{1 / 2}$ \\
\hline P(cH-iPF $)$ & 13.0 \\
P(cH-tBF $)$ & 12.4 \\
P(sB-iPF $)$ & 9.8 \\
P(sB-tBF $)$ & 9.6 \\
P(iP-tBF $)$ & 9.7 \\
P(4mP-iPF $)$ & 9.5 \\
P(4mP-tBF $)$ & 9.4 \\
\hline
\end{tabular}

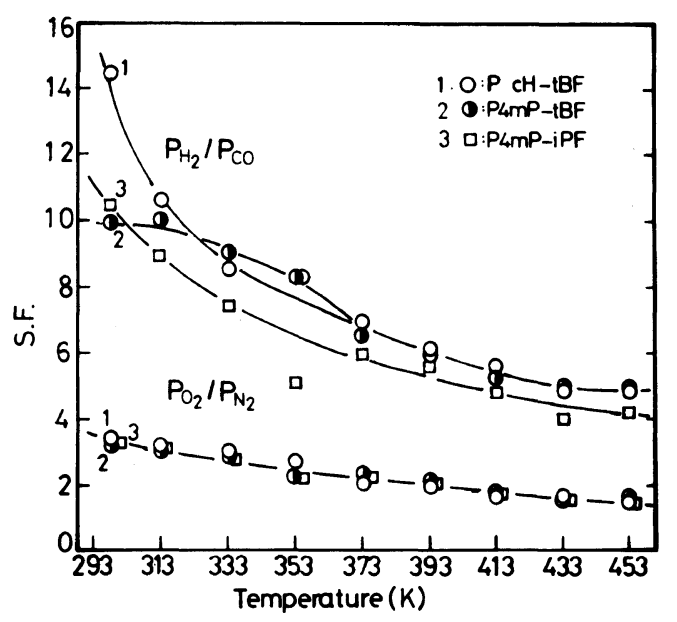

Figure 8. Temperature dependence of separation factors $P_{\mathrm{H}_{2}} / P_{\mathrm{CO}}$ and $P_{\mathrm{O}_{2}} / P_{\mathrm{N}_{2}}$ for poly(dialkylfumarate)s.

PDAFs. The magnitudes of $\delta_{\text {sp }}$ were estimated by the method of Fedors. ${ }^{19}$ Except for PDAFs with cyclohexyl substituent group, the $\delta_{\text {sp }}$ is almost of the same magnitude. The value for poly(alkyl $t$-butylfumarate) is lower than that for poly(alkyl isopropylfumarate). This may be ascribed to an decrease in interaction between molecular chains due to an increase in the number of carbon atoms in alkyl substituents. On the basis of the magnitudes of the solubility parameters, it is expected that the poly(4-methylpentyl $t$-butylfumarate) has the highest permeability among the polymers used in this study. This would agree quite well with the experimental results.

Figure 8 shows the temperature dependences of separation factors of hydrogen to 
carbon monoxide gases, $P_{\mathrm{H}_{2}} / P_{\mathrm{CO}}$, and oxygen to nitrogen gases, $P_{\mathrm{O}_{2}} / P_{\mathrm{N}_{2}}$, in these membranes. With increase in temperature, the magnitude of separation factor decreases. The magnitude of $P_{\mathrm{H}_{2}} / P_{\mathrm{CO}}$ takes the range of 10 to 14 and that of $P_{\mathrm{O}_{2}} / P_{\mathrm{N}_{2}}$, over 3 at $298 \mathrm{~K}$. The decrease in magnitude of separation factor is steep at the $\beta$-relaxation region around $330 \mathrm{~K}$. The magnitude of separation factor also depended on the density of membrane, which reflect the packing state of the molecule. The separation factor for poly(cyclohexyl $t$ butylfumarate) with the highest density among these PDAFs showed the highest permselectivity, especially for $P_{\mathrm{H}_{2}} / P_{\mathrm{CO}}$. The separation factor for oxygen to nitrogen showed high a magnitude below $\beta$-absorption temperature.

\section{CONCLUSIONS}

The permeation behavior of PDAFs with various substituents groups were investigated. The magnitude of permeation coefficient strongly depended on the chemical structure of ester substituents. The magnitude of permselectivity of $\mathrm{H}_{2}$ to $\mathrm{CO}$ was above 10 at $293 \mathrm{~K}$ and decreased above the $\beta$-absorption temperature.

\section{REFERENCES}

1. S. T. Hwang, C. K. Choi, and K. Kammermeyer,
Sep. Sci., 9, 461 (1974).

2. V. T. Stannet, W. J. Koros, D. R. Paul, H. K. Lonsdale, and R. W. Baker, Adv. Polym. Sci., 32, 69 (1979).

3. A. Takizawa, Sen-i Gakkaishi, 40, 229 (1984).

4. W. J. Schell and D. D. Housten, Chem. Progr. Eng., 78(10), 33 (1982).

5. T. Kajiyama, S. Washizu, and M. Takayanagi, $J$. Appl. Polym. Sci., 29, 3955 (1984).

6. A. S. Michaels, W. R. Vieth, and J. A. Barrie, J. Appl. Phys., 34, 13 (1963).

7. W. R. Vieth, J. M. Howell, and J. H. Hsieh, J. Membrane Sci., 1, 177 (1976).

8. K. Toi, G. Morel, and D. R. Paul, J. Appl. Polym. Sci., 27, 2977 (1982).

9. T. Otsu, O. Ito, N. Toyoda, and S. Mori, Makromol. Chem., Rapid Commun., 2, 725 (1981).

10. T. Otsu, H. Minami, N. Toyoda, and T. Yasuhara, Makromol. Chem. Suppl., 12, 133 (1985).

11. K. Yamada, M. Takayanagi, and Y. Murata, Polymer, 27, 1045 (1986).

12. S. B. Choi, A. Takahara, N. Amaya, Y. Murata, and T. Kajiyama, Polym. J., 21, 215 (1989).

13. R. M. Barrer and G. Skirrow, J. Polym. Sci., 3, 549 (1948).

14. T. Kajiyama, Y. Nagata, E. Maemura, and M. Takayanagi, Chem. Lett., 676 (1979).

15. T. Kajiyama, S. Washizu, and M. Takayanagi, $J$. Appl. Polym. Sci., 29, 3955 (1984).

16. S. Washizu, I. Terada, T. Kajiyama, and $\mathbf{M}$. Takayanagi, Polym. J., 16, 307 (1984).

17. J. Brandrup and E. H. Immergut, "Polymer Handbook," 2nd ed, John Wiley, New York, N. Y., 1975, p 111.

18. K. Haraya, K. Obada, T. Hakuda, and $\mathrm{H}$. Yoshitome, Membrane, 11, 48 (1986).

19. R. F. Fedors, Polym. Eng. Sci., 4, 147 (1974). 